\title{
Parameter Optimization in Multi-scale Segmentation of High Resolution Remotely Sensed Image and Its Application in Object-oriented Classification
}

\author{
Lan Zheng \\ College of Geography \\ Fujian Normal University \\ Fuzhou 350007, China \\ Fujian Provincial Engineering Research Center for Monitoring and Assessing Terrestrial Disasters \\ Fuzhou 350007, China
}

\begin{abstract}
With the widely use of high resolution remotely sensed image, the methodology of object-oriented classification emerged and became an active research area. Two basic steps of objectoriented classification method is segmentation and classification, and the effect of segmentation will directly affect the accuracy of classification. An optimal parameters method of multi-scale segmentation was described in this paper with the SPOT-5 high spatial resolution image of Pingtan Island in Fujian province, $P$. R. China. The cross validation technology was employed to compared the optimal value of the $\mathbf{4}$ parameters as scale, shape, color and compactness. The classification results of the traditional pixel-based supervised classification method and the object-oriented classification method were also checked using error matrix to qualify which method would be better. The result shows that object-oriented classification can make full use of spectral information, geometric structure and texture features of the image, and it has a better prospect in classification of the high spatial resolution remote sensing image.
\end{abstract}

Keywords-segmentation; parameter optimization; objectoriented classification; high resolution remotely sensed image

\section{INTRODUCTION}

As state-of-the-art technology, the classification method of remotely sensed image can be cataloged into the traditional pixel-based method and object-oriented method[1]. The former is mainly considered about the spectral characteristics, while ignoring the other informations that lead to a result of salt and pepper phenomenon of classification and the accuracy is relatively low. And because of the phenomenon of " the same spectrum for differentsurface features" and " the different spectrums for the same features" emergenced, it is difficult to obtain satisfactory results[2]. While object-oriented classification method breaks through the limitations of traditional classification, the image will be segmented into a homogeneous object instead of pixels as the basic unit of image classification[3]. Not only the spectral features of the image are used, but also the shape, geometry, texture and context information of the high resolution image are fully employed[4-6].

Image segmentation is an important technology in objectoriented classification, but it cannot be measured by a quantitative index. So this paper will choose the optimal parameters of multiresolution segmentation by orthogonal experiments and applied it in SPOT-5 image of Pingtan Island. The land use types were extracted and the advantages of objectoriented classification were verified by quantitative evaluation.

\section{STUDY AREA AND DATA}

The study area is Pingtan Island in Fujian Province, which is located at latitude $25^{\circ} 15^{\prime}-25^{\circ} 45^{\prime}$ ', longitude $119^{\circ} 32^{\prime}-120^{\circ} 10^{\prime}$. This island has an area of 267.13 square kilometers, the main land use types are forests, water, roads, residential areas, cultivated land, sea farms and others.

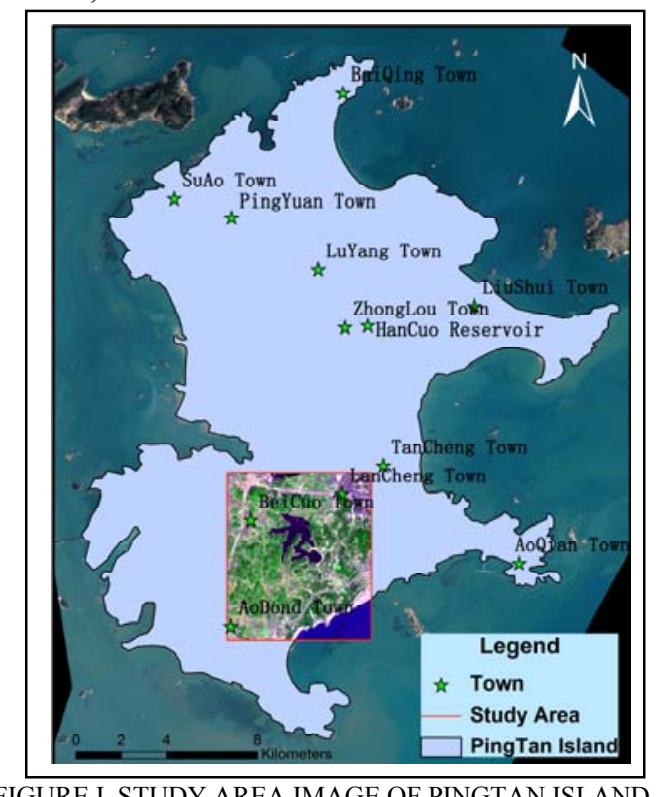

Study area selected Fujian Pingtan Island image of the SPOT-5 acquied on December 02, 2008, using $2.5 \mathrm{~m}$ resolution of panchromatic and $10 \mathrm{~m}$ resolution of multi-spectral for experiment. Firstly, the data should do geometric correction and atmospheric correction,then fusion panchromatic and multi spectral images, which can not only preserves the geometrical 
features and resolution of high resolution images, but also increases the abundant spectral features of the multi spectral images.Finally, clipping a rectangular area of Pingtan island as a sample of the experiment. (As shown in Figure 1)

\section{PARAMETER OPTIMIZATION OF MULTI-SCALE SEGMENTATION}

\section{A. Overview of Multi-scale Segmentation}

Object-oriented classification is based on the image spectrum, texture, structure and other characteristics to segment the image into objects rather than pixel-based unit with a certain homogeneity standard[7-8]. Pixels in each object have some " Homogeneity" on the spectral and spatial structure that can effectively avoid the " salt and pepper phenomenon" [9], so the result has a better overall performance.

At present, the first object-oriented image analysis software in commercial remote sensing is eCognition. To some extent, the software can improve the efficiency and accuracy which the traditional classification method can not take into account. And its core processes include both segmentation and classification, segmentation is the basis step which will directly affect the accuracy of the classification. Therefore, it is very important to select the appropriate parameters[10-11].

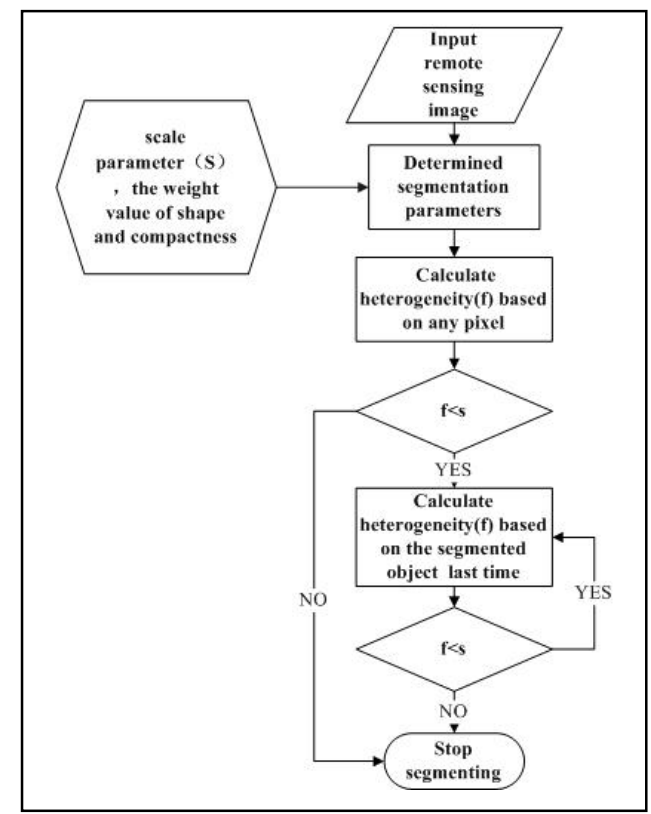

FIGURE II. SEGMENTATION PROCESS BASED ON HETEROGENEITY

Multiresolution segmentation is the most common method for object-oriented. It is a combination of spectral, shape and texture information of the region merging technology from bottom to top[12]. It is achieved by calculating the object heterogeneity, when heterogeneity is less than the setting threshold, then merge adjacent pixels or small objects to ensure a minimum average heterogeneity between objects and maximum internal homogeneity under the premise, gradually merging into large objects.And finally stop merge until the segmentation of the object heterogeneity is greater than or equal to the setting threshold[13]. (As show in Figure 2)
Image segmentation of heterogeneity mainly use the heterogeneity of spectral and shape, by calculating the comprehensive characteristics value of each band's spectral and shape weight value, then calculated the sum of all bands' characteristic values according to each band's weight value[14$15]$.

$$
h=w_{1} \cdot h_{\text {color }}+\left(1-w_{1}\right) \cdot h_{\text {shape }}
$$

In equation (1), $\mathrm{w}_{1}$ is the weigh value, $0 \leq \mathrm{w}_{1} \leq 1 ; \mathrm{h}_{\text {color }}$ is Spectral heterogeneity and $h_{\text {shape }}$ is shape heterogeneity.

\section{B. Segmentation Parameters}

1) Scale parameter: In multiscale segmentation, the selection of the scale parameter is very important, it determines the maximum heterogeneity among different objects. The larger the scale parameter is, the larger object area is generated, and the number of objects is fewer at the same time[16]. Generally, it will be better to choose a bigger scale parameter by ensuring that the boundary of the object can be separated[17]. Through the experiment of Figure 3, it is found that segmented objects are too broken when the scale parameter is less than 20 , it reduce the heterogeneity between objects and the number of polygons, which will decrease the classification accuracy; And when the scale parameter is more than 100 , it will result in different types of land use mixed together, unable to extract the exact boundary so that it will be conducive for classification. So the scale parameter choice is between 20-100 in the following experiments.

2) Shape parameter and color parameter: The weight value sum of shape parameter and color parameter is 1 . It is suitable to use high weight of color values if spectral characteristics of features are obvious, while high weight of shape values are more suitable for regular featureas[18]. Since the most important information in the image is spectral information, So we should choose higher weight of the spectral characteristics as far as possible when meeting with the shape standard of the premise[17]. Through experiments, the influence of different shape parameters on the segmentation is viewed in the case of scale parameter and compactness parameter are fixed. We can inferred from Figure 4 that the smaller shape parameter is, the more irregular of segmented objects will be. While the shape parameter is larger, the difference of area between segmented objects is smaller, and more regular, but it can not reflect the real situation of the surface features according to the spectral characteristics.

3) Compactness parameter and smooth parameter: The shape parameter is composed of the compactness and smoothness, and their sum of weight is 1 . Smooth parameter refers to the smoothness of the segmented objects' boundary, the higher weight shows the more smooth boundary of the objects; Compactness parameter refers to the degree of compactness in each segmented object, the higher the weight, the more compactness of internal object. Through experiments, 
the influence of different compactness parameters on the segmentation is viewed in the case of scale parameter and shape parameter are fixed. We can inferred from Figure 5 that the smaller the compactness, the more broken of the segmented objects.

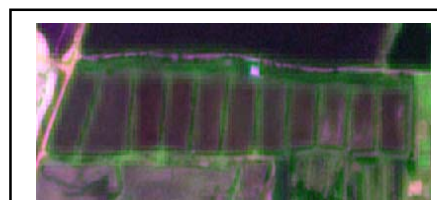

(a) Original image

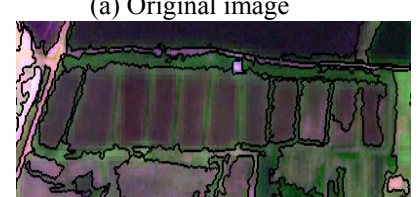

(c) Scale 80

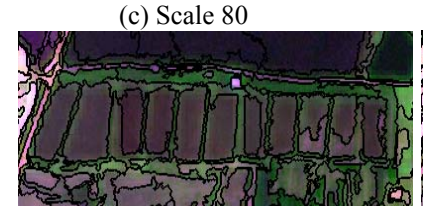

(e) Scale 40

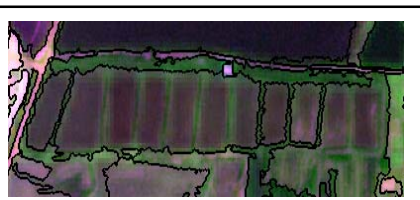

(b) Scale 100

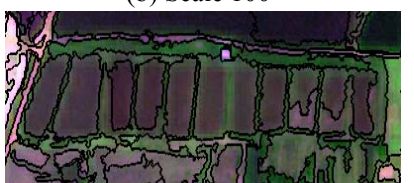

(d) Scale 60

(f) Scale 20

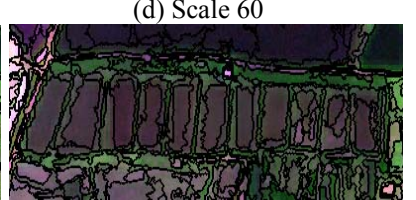

FIGURE III. EFFECT OF SCALE PARAMETER

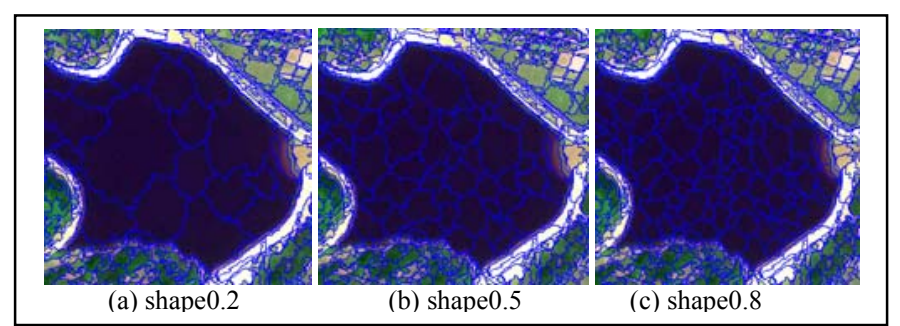

FIGURE IV. EFFECT OF SHAPE PARAMETER

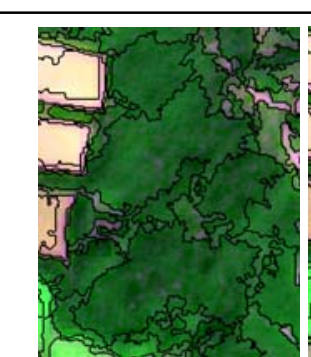

(a) compactness 0.8

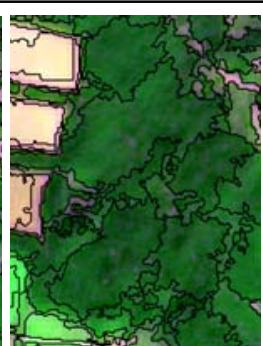

(b) compactness 0.5

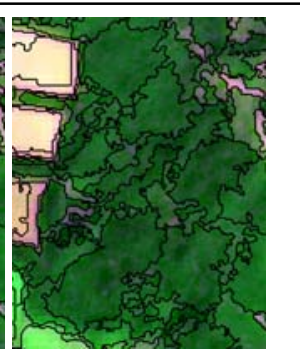

(c) compactness 0.2
FIGURE V. EFFECT OF COMPACTNESS PARAMETER

According to the experiments above, the scale parameter is chosen to be between 20 and 100 , neither will the surface features mixed too much, nor will the objects be too broken. We can choose scale, shape and compactness parameter to represent all parameters. Then three representative levels were selected to test for these three parameters. The levels of scale parameter select $30,90,60$, and the shape parameter is 0.2 , $0.8,0.5$, and $0.2,0.5,0.8$ for compactness parameter.

\section{Cross Validation}

For the three parameters and three levels of testing in this study, there are usually two methods to optimize the parameter. One is comprehensive test, with three levels of the three parameters to do the whole arrangement, a total of 27 tests need to done to select the best combination of segmentation parameters. Although this method is very comprehensive, but the number of tests is too much and it requires a lot of time. what'more, it is even impossible to achieve in the multiparameter and multi-level tests. The other method is the simple contrast method, that is, change one of the parameters with other parameters fixed, so that the optimal level of each parameter will be determined. Taking the data of this study as an example, firstly fixed shape parameter and compactness parameter, and the optimal level of scale parameter will be selected by comparing the effect of different scale parameter levels. In the same way, the optimal level of shape parameter and compactness parameter can also be selected as well. Although this method reduces the number of tests, but the representation is not good enough and there still have certain errors in the test. Combining these two methods above, this study decided to use the method of orthogonal test to optimize the parameters of multiresolution segmentation.

TABLE I. DEVIATION ORTHOGONAL TABLE

\begin{tabular}{|c|c|c|c|c|}
\hline No. & Scale & Shape & Compactness & Deviation \\
\hline 1 & 30 & 0.2 & 0.2 & 0.0014 \\
\hline 2 & 30 & 0.5 & 0.5 & 0.0214 \\
\hline 3 & 30 & 0.8 & 0.8 & 0.0172 \\
\hline 4 & 60 & 0.2 & 0.5 & 0.0248 \\
\hline 5 & 60 & 0.5 & 0.8 & 0.1206 \\
\hline 6 & 60 & 0.8 & 0.2 & 0.1946 \\
\hline 7 & 90 & 0.2 & 0.8 & 0.3865 \\
\hline 8 & 90 & 0.5 & 0.2 & 0.7504 \\
\hline 9 & 90 & 0.8 & 0.5 & 1.2136 \\
\hline
\end{tabular}

Cross validation technology is a kind of method that selected the representative tests to replace the comprehensive tests. And gain a comprehensive test case by analyzing the results of a representative experiments. Therefore, the selection of the representative test is the key of cross validation technology[19]. The cross validation table is a kind of form to arrange the representative test and data analysis. As for the three levels of three parameters, the traditional method needs to carry out 27 tests, while using the cross validation table to arrange tests requires only 9 . Such a method, to some degree, can not only reduces the number of tests, but also has representative. Table 1 is a Orthogonal tables for the test.

First of all, select 4 representative land use types to vector, as shown in Figure 6. The standard of judging the segmentation effect by using each polygon'area. Then, do the multiresolution segmentation tests in eCognition according to the orthogonal table arrangement in Table 1 and compared these 9 tests with the vectors. Finally, the general linear analysis of the single variable with SPSS is obtained, which is shown in Table 2. In equation (2), (3), (4), S is the sum of the area for 4 kinds of land use, $S_{i}$ is the sum of 4 kinds of land use area of 9 experiments in Table 1, D is the deviation degree of the standard polygon and the segmented objects. 


$$
S=\sum_{k=a}^{d} S_{k}
$$

$$
S_{i}=\sum_{j=a}^{d} S_{i j}
$$

$$
D=\left|S_{i}-S\right| / S
$$

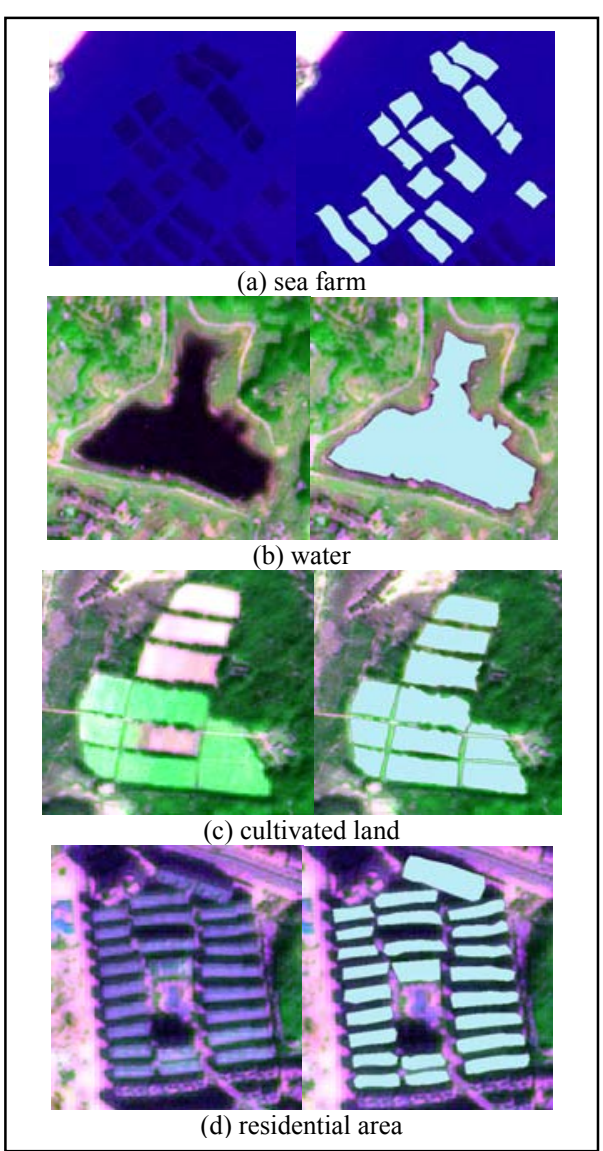

FIGURE VI. INTERPRETATION OF DIFFERENT LAND USE

TABLE II. SingLE FACTOR STATISTICS (DEVIATION)

\begin{tabular}{|c|c|c|c|c|}
\hline & Mean & Std.Error & $\begin{array}{c}\text { Lower } \\
\text { Bound }\end{array}$ & $\begin{array}{c}\text { Upper } \\
\text { Bound }\end{array}$ \\
\hline scale & & & & \\
\hline 30 & 0.013 & 0.127 & -0.532 & 0.559 \\
\hline 60 & 0.113 & 0.127 & -0.432 & 0.659 \\
\hline 90 & 0.784 & 0.127 & 0.238 & 1.329 \\
\hline Shape & & & & \\
\hline 0.2 & 0.138 & 0.127 & -0.408 & 0.683 \\
\hline 0.5 & 0.298 & 0.127 & -0.248 & 0.843 \\
\hline 0.8 & 0.475 & 0.127 & -0.071 & 1.021 \\
\hline Compactness & & & & \\
\hline 0.2 & 0.316 & 0.127 & -0.230 & 0.861 \\
\hline 0.5 & 0.420 & 0.127 & -0.126 & 0.966 \\
\hline 0.8 & 0.175 & 0.127 & -0.371 & 0.720 \\
\hline
\end{tabular}

TABLE III. DEVIATION ORTHOGONAL TABLE

\begin{tabular}{|c|c|c|c|c|}
\hline No. & Scale & Shape & Compactness & Deviation \\
\hline 1 & 20 & 0.1 & 0.7 & 0.0257 \\
\hline 2 & 20 & 0.2 & 0.8 & 0.0207 \\
\hline 3 & 20 & 0.3 & 0.9 & 0.0117 \\
\hline 4 & 30 & 0.1 & 0.8 & 0.0076 \\
\hline 5 & 30 & 0.2 & 0.9 & 0.0166 \\
\hline 6 & 30 & 0.3 & 0.7 & 0.0216 \\
\hline 7 & 40 & 0.1 & 0.9 & 0.0142 \\
\hline 8 & 40 & 0.2 & 0.7 & 0.0134 \\
\hline 9 & 40 & 0.3 & 0.8 & 0.0156 \\
\hline
\end{tabular}

TABLE IV. Single Factor Statistics (DEVIATION)

\begin{tabular}{|c|c|c|c|c|}
\hline & Mean & Std.Error & $\begin{array}{c}\text { Lower } \\
\text { Bound }\end{array}$ & $\begin{array}{c}\text { Upper } \\
\text { Bound }\end{array}$ \\
\hline scale & & & & \\
\hline 20 & 0.019 & 0.005 & -0.001 & 0.040 \\
\hline 30 & 0.015 & 0.005 & -0.005 & 0.036 \\
\hline 40 & 0.014 & 0.005 & -0.006 & 0.035 \\
\hline Shape & & & & \\
\hline 0.1 & 0.016 & 0.005 & -0.004 & 0.036 \\
\hline 0.2 & 0.017 & 0.005 & -0.003 & 0.037 \\
\hline 0.3 & 0.016 & 0.005 & -0.004 & 0.037 \\
\hline Compactness & & & & \\
\hline 0.7 & 0.023 & 0.005 & -0.006 & 0.034 \\
\hline 0.8 & 0.015 & 0.005 & -0.006 & 0.035 \\
\hline 0.9 & 0.020 & 0.005 & -0.001 & 0.040 \\
\hline
\end{tabular}

Table 2 shows the average value of scale parameter, shape parameter and compactness parameter at different levels, the smaller deviation is, the better segmentation effect will have; Through intuitive analysis, the levels of the pros and cons for each parameter order is: scale parameter $30>60>90$, shape parameter $0.2>0.5>0.8$, compactness parameter $0.8>0.2>$ 0.5 . It can be concluded that a combination of better segmentation is 30 for the scale parameter, 0.2 for shape parameter and 0.8 for compactness.

In order to determine the optimal combination, we use the optimal combination $(30,0.2,0.8)$ which was obtained by the former tests as basis, and selected three levels of the three parameters to test. The levels of scale parameter select 20,30, 40 , the shape parameter is $0.1,0.2,0.3$, and $0.7,0.8,0.9$ for compactness parameter. The test and analysis are carried out by using the orthogonal table in Table 3, and the general linear analysis of the single variable is used according to the same test procedure. Results as shown in Table 4, determine the best combination of segmentation is 40 for scale parameter, 0.1 for shape parameter and 0.7 for compactness parameter by the analysis of variance.

\section{OBJECT-ORIENTED CLASSIFICATION AND ACCURACY ASSESSMENT}

\section{A. Classification Rules}

Water class is mainly through the establishment of water normalized index NDWI to extract, but it will mix the shadow of many buildings and forests if only using NDWI to classify, so it is necessary for other constraints to filter the non water 
type. Because the consistency of the water class is relatively high, and the segmented polygon is relatively large, so the number of area pixels can be used as another limiting condition. The final classification rules for water class is NDWI $\geq 0.4$ and area $\geq 2000 \mathrm{pxl}$.

The forests class may be determined by establishing the normalized vegetation index NDVI rules; The road is usually a long strip, and the main distinction with other types of features lies in its larger length/width value, we can also extract features by the value of density. So the road's final classification rule is length/width $\geq 4$. The brightness of the residential areas is relatively high, so we can use it as a limited rule to extract residential areas, and The final classification rules for residential areas is $80 \leq$ brightness $\leq 150$.

\section{B. Classification Results}

Figure 7 is the results for maximum likelihood classification and object-oriented classification. In this study, the results of these two methods of classification were taken 200 random points, and combined with the real properties of the image to calculate the error matrix, Tables 6 and 7 are error matrix for these two methods respectively.
As shown in Table 6, the overall accuracy of the maximum likelihood classification is $77.5 \%$, and the kappa is 0.7118 . The user's accuracy of cultivated land, residential area and road were $62.96 \%, 63.16 \%$ and $68.57 \%$ respectively, which has a higher misclassified phenomenon. However, the producer accuracy of residential areas, bare land and forests were $77.08 \%, 68.57 \%$ and $56.25 \%$, which leads to leakage phenomenon. Only 34 pixels in the classification of cultivated land are correctly classified. This is because the brightness of cultivated land and residential areas are relatively high, and spectral information of cultivated land and forests are similar, so it confused with forests and residential areas; And the road is easily confused with bare land on both sides; The shadow of residential areas is likely to be misclassified by forests.

The overall accuracy of object-oriented classification is $91.5 \%$, kappa is 0.8927 . The extraction is more continuous for road class compared to the maximum likelihood classification. And the accuracy is greatly improved, indicating that the classification results of study area is satisfied. The phenomenon of salt and pepper has been improved to some extent, and it is helpful for the later vectoring and mapping.

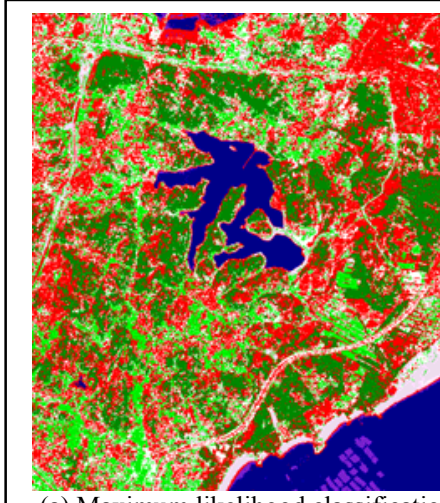

(a) Maximum likelihood classification

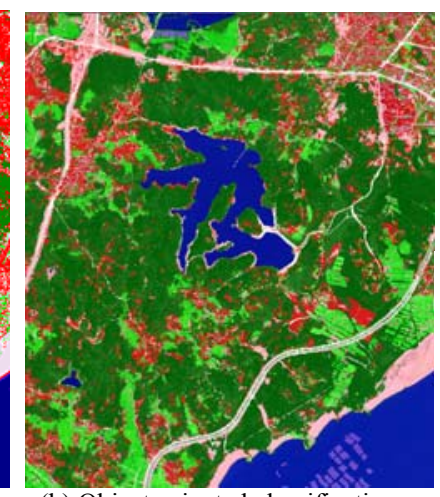

(b) Object-oriented classification

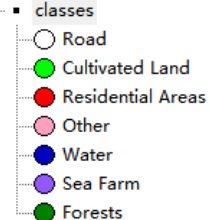

(c) Legend

FIGURE VII. COMPARISON BETWEEN PIXEL AND OBJECT-ORIENTED CLASSIFICATION METHOD

TABLE V. ERROR MATRIX OF MAXIMUM LIKELIHOOD METHOD

\begin{tabular}{|c|c|c|c|c|c|c|c|c|c|}
\hline Type & Forests & Water & $\begin{array}{c}\text { Sea } \\
\text { farms }\end{array}$ & $\begin{array}{c}\text { Cultivated } \\
\text { land }\end{array}$ & $\begin{array}{l}\text { Residential } \\
\text { areas }\end{array}$ & Roads & Other & $\begin{array}{l}\text { Row } \\
\text { total }\end{array}$ & User accuracy\% \\
\hline Forests & 57 & 0 & 0 & 1 & 3 & 1 & 0 & 62 & 91.94 \\
\hline Water & 0 & 16 & 0 & 0 & 0 & 0 & 0 & 16 & 100.00 \\
\hline Sea farms & 0 & 1 & 3 & 0 & 0 & 0 & 0 & 4 & 75.00 \\
\hline Cultivated land & 10 & 0 & 0 & 34 & 6 & 0 & 4 & 54 & 62.96 \\
\hline Residential areas & 8 & 1 & 0 & 1 & 24 & 0 & 1 & 35 & 68.57 \\
\hline Roads & 2 & 0 & 0 & 1 & 2 & 12 & 2 & 19 & 63.16 \\
\hline Other & 1 & 0 & 0 & 0 & 0 & 0 & 9 & 10 & 90.00 \\
\hline Col total & 78 & 18 & 3 & 37 & 35 & 13 & 16 & 200 & \\
\hline Producer accuracy $\%$ & 73.08 & 88.89 & 100.00 & 91.89 & 68.57 & 92.31 & 56.25 & & \\
\hline
\end{tabular}


TABLE VI. ERROR MATRIX FOR OBJECT-ORIENTED CLASSIFICATION

\begin{tabular}{|c|c|c|c|c|c|c|c|c|c|}
\hline Type & Forests & Water & $\begin{array}{c}\text { Sea } \\
\text { farms }\end{array}$ & $\begin{array}{c}\text { Cultivated } \\
\text { land }\end{array}$ & $\begin{array}{l}\text { Residential } \\
\text { areas }\end{array}$ & Roads & Other & $\begin{array}{l}\text { Row } \\
\text { total }\end{array}$ & User accuracy\% \\
\hline Forests & 57 & 0 & 0 & 0 & 0 & 0 & 1 & 58 & 98.27 \\
\hline Water & 0 & 17 & 0 & 0 & 0 & 0 & 0 & 17 & 100.00 \\
\hline Sea farms & 0 & 1 & 5 & 0 & 0 & 0 & 0 & 6 & 83.33 \\
\hline Cultivated land & 2 & 0 & 0 & 47 & 2 & 0 & 1 & 52 & 90.38 \\
\hline Residential areas & 3 & 0 & 0 & 1 & 32 & 0 & 0 & 36 & 83.33 \\
\hline Roads & 1 & 0 & 0 & 0 & 1 & 15 & 1 & 18 & 83.33 \\
\hline Other & 1 & 0 & 0 & 1 & 0 & 1 & 10 & 13 & 76.92 \\
\hline Col total & 64 & 18 & 5 & 49 & 35 & 16 & 13 & 200 & \\
\hline Producer accuracy $\%$ & 89.06 & 94.44 & 100.00 & 95.91 & 91.43 & 93.75 & 76.92 & & \\
\hline
\end{tabular}

\section{CONCLUSIONS}

This paper is based on the object-oriented classification to extract the information of features from Pingtan Island. Object-oriented classification can not only takes advantage good of the spectral information of the image, but also combines the texture, structure and shape effectively. It use high homogeneity pixels that merged into target objects as basic unit, which react more integrity and homogeneity compared to traditional pixels[20-21]. what's more, in a certain extent, it solve the high-resolution remotely sensed images with " the same spectrum for differentsurface features" and " the different spectrums for the same features" phenomena and improve the accuracy of classification[22].

The main two steps of object-oriented classification are segmentation and classification. The effect of segmentation will directly affect the accuracy of classification[23]. Because there is no quantitative index as a standard to judge the effect of high resolution image segmentation, and the visual judgement often has certain subjective error. Therefore, this paper adopts cross validation technology to optimize the different parameters. Finally, applied the optimal segmentation parameters to the study area. The optimal parameters can be changed according to different images and different features, so it needs to search for appropriate parameters through continuous experiments. Classification is mainly considered about the establishment of functions to extract the different feature types, the function of the classification rules and threshold also needs redefining in different images and different feature classification sequence.

In conclusion, the object-oriented classification is more suitable for high-resolution image compared to pixel-based method and have obvious advantages, which can make full use of the spectrum, texture and other information to achieve better classification results. And the universal applicability of object-oriented classification will be the future direction for high resolution remote sensing image classification.

\section{ACKNOWLEDGMENT}

This work was funded by Fujian Bureau of Surveying, Mapping and Geoinformation (Grant No. 2013S17) and Key Project of the Department of Science and Technology of Fujian Province, China (Grant No. 2012Y4001).

\section{REFERENCES}

[1] A. M. Dean, and G. M. Smith, "An Evaluation of Per-parcel Land Cover Mapping Using Maximum Likelihood Class Probabilities," International Journal of Remote Sensing, 14th ed., vol.24.,2003,pp.2905-2920.

[2] Q. L. Tan, and J. J. Gao, "Object-oriented Classification in Extracting Buildings of High-resolution Multi-spectral Image," Engineering of Surveying and Mapping, 4th ed., vol.19.,2010,pp.30-32.

[3] R. M. Hu, M. Wei, and C. B. Yang, "Comparison of Pixel and Objectoriented Classification Based on SPOT-5 Remote Sensing Image," Remote Sensing Technology and Application, 3rd ed., vol.27.,2012,pp.336-370.

[4] A. Su, J. Li, and Y. Chan, "Textural and Local Spatial Statistics for the Object-oriented Classification of Urban Areas Using High Resolution Imagery," International Journal of Remote Sensing, 11th ed., vol.29.,2008,pp.3105-3117.

[5] Y. Qian, and G. Peng, "Object-based Detailed Vegetation Classification With Airborne High Spectrum Resolution Remote Sensing Imagery," Photogram Metric Engineering \& Remote Sensing, 7th ed., vol.72.,2006,pp.799-811.

[6] A. Lobo, O. Chic, and A. Casterad, "Classification of Mediterranean Crops with Multi-sensor Data Per-pixel Versus Per-object Statistics and Image Segmentation," International Journal of Remote Sensing, vol.17.,1996,pp.2358-2400.

[7] X. X. Sun, J. X. Zhang, and Z. J. Liu, "Object-oriented Classification Method to Extract the River and the Road From IKONOS Panchromatic Image," Science of Surveying and Mapping, 1st ed., vol.31.,2006,pp.6263.

[8] Y. Zhong, J. Zhao, and L. Zhang, "A Hybrid Object-Oriented Conditional Random Field Classification Framework for High Spatial Resolution Remote Sensing Imagery," Geoscience and Remote Sensing, IEEE Transactions on,11th ed., vol.52.,2014,pp.7023-7035.

[9] Z. P. Sun, W. M. Shen, and B. Wei, "Object-oriented Land Cover Classification Using HJ-1 Remote Sensing Imagery," Science China. Earth Sciences, vol.53.,2010,pp.33-34.

[10] S. Ryherd, and C. E. Woodcock, "Combining Spectral and Texture Data in the Segmentation of Remotely Sensed Images," Photogrammetric Engineering and Remote Sensing, 2nd ed., vol.62.,1996,pp.181-194.

[11] B. Kartikeyan, A. Sarkar, and K. Majumder, "A Segmentation Approach to Classification of Remote Sensing Imagery," International Journal of Remote Sensing, 9th ed., vol.19.,1998,pp.1695-1709.

[12] M. A. Baatz, and A. Schaepe, "Multir Esolution Segmentation An Optimization Appr Oach for High Quality Multiscale Image Segment Ation," Proceeding from the An2gewandte Geogr Aphische Informationsver Arbeitung XII,Salzburg 2000. Karlsruhe: H erber t Wichmann Verlag, 12223.

[13] Q. L. Tan, Z. J. Liu, and W. Shen, "Multi Resolution Segmentation Based on Object-Oriented Classification of Remote Sensing Image," Journal of Beijing Jiaotong University, 4th ed., vol.31.,2007,pp.111-114.

[14] G. J. Zhou, and C. Z. Yuan, "Research on Geographical Conditions Monitoring Based on Object-oriented Classification Technology," Geomatics \& Spatial Information Technology, 6th ed., vol.37.,2014,pp.162-164. 
[15] S. H. Lowe, and X. Guo, "Detecting an Optimal Scale Parameter in Object-Oriented Classification," Selected Topics in Applied Earth Observations and Remote Sensing, IEEE Journal of, 4th ed., vol.4.,2011,pp.890-895.

[16] K. S. Luo, R. D. Li, and B. R. Chang, "Survey Method of Large Scale Land Cover Based on Object Classification Technology," Journal of University of Chinese Academy of Science, 6th ed., vol.30.,2013,pp.770-777.

[17] W. H. Wang, and M. He, "Multiresolution Segmentation of Objectoriented Classification in Extracting Land Use," Science of Surveying and Mapping, 4th ed., vol.36.,2011,pp.306-310.

[18] M. M. Zhang, and G. H. Yao, "Information Extraction Technology of Object-oriented Classification in High-resolution Remote Sensing Image," Geospatial Information, 1st ed., vol.11.,2013,pp.89-94.

[19] R. J. Liu, Y. W. Zhang, and C. W. When, "Orthogonal Experimental Design and Analysis Research," Experimental Technology and Management, 9th ed., vol.27.,2010,pp.52-55.

[20] A. S. Laliberte, A. Rango, and K. M. Havstad, "Objectoriented Image Analysis for Mapping Shrub Encroachment From 1937 to 2003 in Southern NewMexico," Remote Sensing of Environment, vol.93.,2004,pp.198-210.

[21] J. Schiewe, L. Tufte, and M. Ehlers, "Potential and Problems of MultiScale Segmentation Methods in Remote Sensing," GeoBITPGIS, vol.6.,2001,pp.34-39.

[22] W. Su, J. Li, and Y. H. Chen, "Urban Land Cover of Object-oriented Classification Based on Multi Scale Image Segmentation -- A Case Study of Malaysia City Center District," Journal of Remote Sensing, 4th ed., vol.11.,2007,pp.521-529.

[23] J. C. Guo, P. J. Li, and X. Xiao, "Multi Scale Segmentation Method for High Resolution Multispectral Images," Acta Scientiarum Naturalium Universitatis Pekinensis, 2nd ed., vol.45.,2009,pp.306-310. 